\title{
Aplicación de Modelos de Inventarios en una Cadena de Abastecimiento de Productos de Consumo Masivo con una Bodega y M Puntos de Venta
}

* Ph.D. Profesor Escuela de Ingeniería Industrial y Estadística - Facultad de Ingeniería - Universidad del Valle, Santiago de Cali, Colombia.

E-mail:cjvidal7@calipso.com.co

** Ingeniero Industrial - Estudiante de la Maestría en Ingeniería, Área de Énfasis, Ingeniería Industrial - Facultad de Ingeniería

- Universidad el Valle, Santiago de Cali, Colombia

E-mail:jucelo61@yahoo.com.ar

*** Caja de Compensación Familiar del Valle del Cauca COMFAMDI, Santiago de Cali, Colombia

E-mail:fernandocontreras@comfandi.com.co

Fecha de recepción: Abril 30 de 2004

Fecha de aprobación: Septiembre 14 de 2004

\author{
Carlos Julio Vidal Holguín* \\ Julio César Londoño Ortega** \\ Fernando Contreras Rengifo***
}

\section{RESUMEN}

Uno de los problemas más complejos que afectan las empresas industriales y comerciales locales es la administración y control de los inventarios. Es muy frecuente escuchar el problema del desbalanceo de inventarios: "¿Por qué será que siempre se tiene mucho de lo que casi no se vende y hay faltantes de lo que sí rota?" En este artículo se abordarán las causas de este problema y sus posibles soluciones, especialmente en cadenas de suministro con una bodega y múltiples puntos de venta (OneWarehouse MRetailer Problem). Se discutirán diversas técnicas de control de inventarios para este sistema, concentrándose también en sus 
aspectos logísticos generales, ilustrando con la aplicación real en una empresa comercializadora de productos de consumo masivo. Los impresionantes resultados obtenidos evidencian un excelente potencial de aplicación de estos modelos en empresas comerciales o industriales interesadas en basar sus procesos de toma de decisiones en la Investigación de Operaciones. Estos resultados motivan el continuo desarrollo de proyectos de investigación aplicada en las organizaciones de la región.

Palabras Clave: Control de Inventarios, OneWarehouse M-Retailer Problem, Sistemas de inventarios multi-escalón, Inventarios de productos de consumo masivo.

\section{ABSTRACT}

Inventory control and management is one of the most complex problems that our industrial and commercial sectors face. Companies usually deal with the inventory imbalance problem: "¿Why is it the case that almost always there is a lot of low-volume products, while high-volume products frequently present stockouts?" The causes of this problem and their possible solutions are addressed in this paper, especially in supply chains with Onewarehouse and $M$ retailers (OneWarehouse MRetailer Problem). Several inventory control techniques for this system are discussed, with emphasis on its general logistics characteristics, illustrated with a real case from a supply chain of products of massive consumption. The impressive results that were obtained suggest excellent opportunities for the application of these models in industrial and commercial organizations interested in adopting Operations Research techniques in their decision making processes. These results encourage the continuous development of applied research projects on this area in local organizations.

Keywords: Inventory Control, One-Warehouse MRetailer Problem, Multi-Echelon Inventory Systems, Inventory control of massive consumption products.

\section{INTRODUCCIÓN ${ }^{1}$}

El control y administración de inventarios es uno de los temas más apasionantes en Logística y una de las principales aplicaciones prácticas de la Investigación de Operaciones (IO). Es muy común escuchar a los administradores, gerentes y analistas de Logística afirmar que uno de sus principales problemas es la administración de los inventarios. El problema típico es la existencia de excesos y de faltantes: "Siempre se tiene demasiado de lo que no se vende, y muchos agotados de lo que sí tiene volumen de ventas." Lo interesante de este problema es que ocurre prácticamente en cualquier empresa del sector industrial o comercial, especialmente en las organizaciones locales. Se presentarán sus causas y las posibles estrategias para resolverlo o al menos atenuarlo.

Las causas fundamentales para la necesidad del mantenimiento de inventarios en cualquier empresa son, inicialmente, el desfase que existe entre la demanda de los consumidores y la producción o suministro de dichos productos $y_{\text {, }}$ principalmente, las fluctuaciones aleatorias de la demanda y de los tiempos de reposición en la cadena de suministro. Las estrategias más comunes para manejar estas fluctuaciones son el mejoramiento de la calidad de la información, el mantenimiento de inventarios de seguridad y la colaboración en la cadena de abastecimiento.

La primera estrategia tiene que ver con la obtención y transmisión de información precisa y en tiempo real sobre la demanda en los puntos de consumo y con la correcta identificación de los productos, las cuales se pueden lograr a través de la implementación de tecnologías modernas de información, tales como Sistemas de Administración de Bodegas (WMS), Intercambio Electrónico de Datos (EDI) y otros sistemas automáticos de identificación, como la

1 Adaptado y complementado de Vidal (2001, 2002) 
naciente Identificación por Radiofrecuencia (RFID). La segunda y tercera estrategias pueden incluir, entre otros, algunos de los siguientes puntos:

■ La consolidación de centros de distribución y bodegas;

■ La estandarización de productos para evitar el mantenimiento de inventarios de una gran diversidad de ellos, que sólo difieren en aspectos menores de forma, color, condición, etc. Las características finales del producto pueden ser implementadas en el momento de recibir las órdenes de los clientes; (Principio de posposición de forma)

च La emisión de órdenes conjuntas para diversos grupos de productos con el objeto de balancear su inventario y la consolidación de despachos desde (hacia) diversas localidades, a través de técnicas como el cross-docking; (Principio de posposición de tiempo)

$\square$ El mejoramiento de los sistemas de pronósticos de demanda a través de la adaptación y aplicación de técnicas estadísticas de reconocida eficacia y de combinación de diversas técnicas cualitativas y cuantitativas;

च El mejoramiento de alianzas y de sistemas de comunicación con proveedores y clientes para la reducción del promedio y la variabilidad de los tiempos de reposición y para el compartimiento de la información de demanda a lo largo de la cadena de suministro.

Debido a que las causas que generan la necesidad de mantener inventarios no pueden ser eliminadas totalmente, y a que la inversión en algunas tecnologías de información y planeación avanzada puede resultar muy costosa y de alto riesgo para la mayoría de las organizaciones locales, una de las mejores alternativas puede ser aplicar sistemas eficientes de gestión y control para responder a dichas causas.
El problema en la mayoría de las organizaciones locales radica principalmente en que los inventarios de seguridad y sus correspondientes puntos de reorden o inventarios máximos se determinan exclusivamente con base en el promedio de la demanda, ignorando su variabilidad y la variabilidad de los tiempos de reposición. Por ejemplo, para cierto producto, se podría establecer el inventario de seguridad en "dos semanas de inventario". Esto significa que, en promedio, el inventario de seguridad tardaría aproximadamente dos semanas en agotarse. En realidad, dicho inventario puede durar mucho menos o mucho más de dos semanas. Cuando la variabilidad de la demanda del producto es baja, dos semanas de inventario de seguridad puede ser un exceso en el que se está invirtiendo capital innecesariamente. Por el contrario, si la variabilidad de la demanda del producto es alta, dos semanas de inventario de seguridad puede ser muy poco y ocurrirán agotados frecuentes de dicho producto.

Sólo en algunas ocasiones los inventarios de seguridad y los puntos de reorden (o los inventarios máximos) calculados únicamente con base en la demanda promedio, coinciden con el valor adecuado obtenido como resultado de un análisis estadístico formal. Es un error conceptual grave, por lo tanto, definir inventarios de seguridad de los productos proporcionalmente a su demanda promedio en forma exclusiva. De aquí precisamente provienen los desbalanceos de inventario mencionados anteriormente, lo cual pudo comprobarse empíricamente en el sistema descrito en este artículo. Es sorprendente observar la frecuencia y persistencia con que este error conceptual se comete en las empresas locales.

La clave consiste entonces en liberar capital invertido en inventarios de seguridad de productos con baja variabilidad y distribuirlo en inventarios de seguridad de productos con alta variabilidad. El balance de esta operación es frecuentemente positivo y se puede mejorar 
significativamente el servicio al consumidor sin invertir un peso adicional en inventarios, se puede mantener el servicio actual con mucho menos capital invertido, o incluso se puede diseñar una combinación intermedia de ambos beneficios.

Algunas estrategias para lograr el balanceo de inventarios son:

ఐ La implementación de la clasificación ABC para facilitar la función administrativa del inventario por categorías, incluyendo la depuración periódica del mismo;

च La utilización de sistemas adecuados de pronósticos que permitan el cálculo de los inventarios de seguridad con base en la variabilidad de la demanda y de los tiempos de reposición, de acuerdo con el nivel de servicio deseado. Deben minimizarse las causas frecuentes de errores excesivos en los pronósticos, tales como la selección del modelo matemático inadecuado, la utilización de datos poco confiables y de datos de ventas en lugar de demanda, los sesgos en los pronósticos, la inclusión de datos atípicos y la selección errada del período fundamental del pronóstico;

$\square$ El diseño y/o selección de sistemas de control de inventarios que funcionen en forma coherente con los sistemas de pronósticos anteriores;

$\square \quad$ La consideración de aspectos fundamentales tales como el ciclo de vida del producto, la naturaleza del proceso productivo o comercial bajo estudio y los aspectos financieros relacionados con los inventarios. Es muy común el error, por ejemplo, de medir el desempeño de un sistema de gestión y control de inventarios a través de su rotación solamente y querer mejorarla incluso a costa del nivel de servicio ofrecido al cliente. Por esta razón, se debe mantener una generación continua de indicadores de eficiencia que reflejen en conjunto toda la operación del sistema de control de inventarios, tales como:
- Indicadores de nivel de servicio al cliente, como probabilidad de ocurrencia de faltantes, porcentaje de demanda satisfecha del inventario a la mano (fill rate) y porcentaje de demanda no satisfecha $y / 0$ nivel de órdenes pendientes (backorders);

- El porcentaje de precisión del inventario físico;

- El valor, composición y grado de obsolescencia del inventario;

- Indicadores financieros tales como el porcentaje del inventario pagado, la cobertura del inventario comparada con los beneficios financieros obtenidos por medio de grandes volúmenes de compra o producción, la rentabilidad de productos por cada clase y línea de productos y el retorno sobre la inversión en inventarios;

- La rotación neta del inventario, basada en el inventario promedio pagado (inventario promedio menos promedio de cuentas por pagar) y en las ventas de contado?

Todas las estrategias anteriores enmarcan las condiciones mínimas necesarias para el correcto diseño e implementación de verdaderos sistemas de control de inventarios en cadenas de abastecimiento. El arte de la aplicación de técnicas de $1 O$ en este caso consiste entonces no sólo en el correcto diseño de modelos matemáticos para el control de inventarios, sino también en permitir que las condiciones anteriores puedan darse.

Este trabajo se concentra entonces en una cadena de abastecimiento con una bodega y $M$ puntos de venta (One-Warehouse M-Retailer Problem), tal como se muestra en la Figura 1. En este tipo de cadenas, muchos proveedores suministran decenas o cientos de miles de

\footnotetext{
${ }^{2}$ La rotación neta en días (o el número neto de días de inventario pagado) se calcula como el cociente entre el inventario pagado promedio cada mes y el costo de ventas mensuales, multiplicado por 30 días/mes.
} 
productos, los cuales se almacenan en una bodega central, de donde son despachados hacia M puntos de venta para su comercialización y satisfacción de la demanda de los consumidores. Algunos problemas de gestión de inventarios en este tipo de cadenas se concentran en determinar la cantidad de inventario de cada producto a mantener en la bodega y en los puntos de venta, las políticas de control en la bodega y en los puntos de venta, y la forma de coordinación entre la bodega central y los puntos de venta. Estas características, por lo tanto, hacen que el control de inventarios de este tipo de sistemas sea extremadamente complejo.

De acuerdo con Silver et al. (1998), la complejidad de estos sistemas surge principalmente de la coordinación de las diferentes localidades y la estrecha relación e impacto que una decisión tomada en un punto de la cadena tiene sobre todos los demás puntos de la misma. Axsäter (2000) afirma que se puede esperar que una política de control óptima para un sistema de control de inventarios de múltiples etapas sea considerablemente compleja, incluso en aquellos casos en los cuales la demanda se puede considerar determinística. Silver et al. (1998) sostienen además que la demanda probabilística crea complejidades extremas de modelación en una situación de inventarios en cadenas de suministro.

El conocido efecto látigo ilustra las complejidades mencionadas. En este fenómeno, por más uniforme que sea la demanda en el punto final de la cadena (consumidores), la demanda inducida sobre los puntos de la cadena situados 'aguasarriba' (la bodega central y los proveedores, en este caso) se vuelve en la mayoría de los casos altamente errática, lo que induce altos niveles de inventarios de seguridad, los cuales pueden ser innecesarios en la mayoría de las ocasiones. Como sostienen Lee et al. (1997), existen cuatro factores que ayudan a producir el efecto látigo:

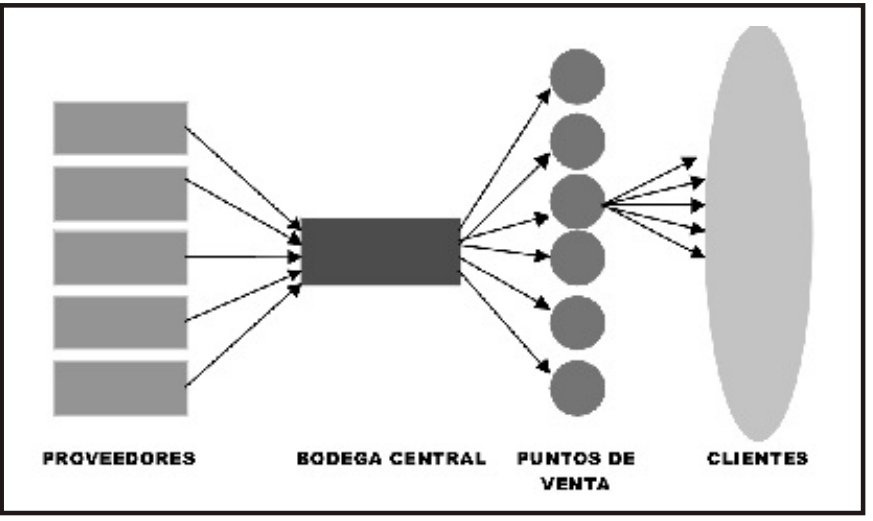

Figura 1. Una cadena de abastecimiento típica con una bodega y $M$ puntos de venta

च La emisión de órdenes anticipadas ante el crecimiento de la demanda, lo que crea un nivel artificialmente alto de la misma;

■ La emisión de órdenes infladas cuando se presenta escasez, para tratar de obtener mayor participación en órdenes compartidas con otros puntos de almacenamiento en la cadena;

च La consolidación de órdenes para tratar de reducir el efecto de los costos fijos;

च La variación de costos de producción y/o compra, lo que induce lotes de manufactura o compra más grandes.

Otras complejidades de un sistema como el mostrado en la Figura 1 pueden incluir las siguientes:

$\square$ Consideraciones de costos y nivel de servicio en las diferentes etapas de la cadena;

$\square \quad$ La definición de tiempo de reposición entre un eslabón y otro de la cadena. Por ejemplo, el tiempo de reposición entre la bodega central y los puntos de venta puede depender de aquel entre los proveedores y la primera;

$\square$ La presencia de órdenes para las cuales no hay disponibilidad completa de productos para todos los puntos de venta y las políticas de asignación en estos casos;

$\square$ La posibilidad de despachos directos desde la bodega central hacia los consumidores, de transferencias entre puntos de venta y el 
manejo de la logística inversa por devoluciones.

Schwarz (1973) fue de los primeros autores en estudiar este tipo de cadenas de suministro y pudo demostrar que, incluso en el caso determinístico para $M$ 3, la forma de la política óptima de control de inventarios puede llegar a ser muy compleja. Muchas aproximaciones a este problema se constituyen en simplificaciones que aproximan el problema complejo mediante situaciones semejantes, pero más simples [Véase, por ejemplo, Axsäter (1993a, 1993b y 2000) y Cheng y Zheng (1997)].

La mayoría de los trabajos para este tipo de cadenas asumen demanda con distribución de Poisson en los puntos de venta y han sido desarrollados para productos de alto valor y lento movimiento [Por ejemplo, Forsberg (1995, 1996), Cohen et al. (1986), Graves (1996) y Axsäter (1998)]. En otros casos, en ambientes productivos con remanufactura (reparación de productos), se asume también demanda de Poisson o procesos de Poisson compuestos. [Véase, por ejemplo, Sherbrooke (1968), con su trabajo pionero denominado METRIC ${ }^{3}$ ] Por otra parte, muchos trabajos asumen que los $M$ detallistas son idénticos [Véase, por ejemplo, Moinzadeh (2002) y Chen y Samroengraja (2000)].

Entre algunas excepciones, que asumen demanda normal en los puntos de venta, se encuentran Axsäter (2000), quien presenta un análisis exacto para un sistema serial con un solo punto de venta, el cual es una extensión del trabajo de Clark y Scarf (1960). Axsäter sostiene que la metodología utilizada puede ser extendida a casos como el que se aborda en este trabajo, como lo presentado en los artículos de Federgruen y Zipkin (1984) para el caso en que la

3 Debido al nombre en Inglés, 'MultiEchelon Technique for Recoverable Item Control' bodega central actúa como un centro de crossdocking, y Matta y Sinha (1995) cuando la bodega central almacena inventario. Otro ejemplo que puede clasificarse dentro de esta categoría es el trabajo de Lee et. al. (2000). La aplicación práctica de los trabajos anteriores, sin embargo, se ve limitada por la necesidad de estimar el costo de faltantes por unidad monetaria y por unidad de tiempo en cada uno de los puntos de venta, para cada uno de los productos que se mantienen en inventario. Además, las múltiples complejidades que un sistema real de una bodega y $M$ puntos de venta presenta, difícilmente podrían incluirse en un modelo integral de control con resultados analíticos.

Axsäter et al. (2002) analizan un sistema con un solo proveedor con capacidad infinita, una bodega central y varios detallistas. El objetivo es encontrar políticas que minimicen los costos de mantenimiento del inventario y de las órdenes pendientes. Para este efecto, se desarrollan dos métodos heurísticos, cuyo comportamiento, de acuerdo con los autores, supera al método tradicional del supuesto del "balance", el cual permite la asignación de inventario negativo a los detallistas. Esto es válido para determinar el punto de reorden de la bodega central y los inventarios máximos de los detallistas, lo que puede causar exceso de inventario en los detallistas.

Más recientemente, han sido publicados algunos trabajos importantes relacionados con el problema de una bodega y M puntos de venta. Özer (2003), por ejemplo, desarrolla un heurístico para establecer políticas de inventario efectivas teniendo en cuenta información de demanda conocida con anticipación en un sistema con un solo proveedor supliendo a una bodega que surte a M puntos de venta. El autor muestra que el tener información avanzada de demanda produce niveles de inventarios y costos relacionados más bajos, lo cual convierte a esta información en sustituto para inventarios y tiempos de reposición 
de seguridad. Otro trabajo de importancia es el presentado por Dong y Lee (2003), el cual reconsidera el trabajo seminal de Clark y Scarf (1960) en un sistema serial multi-eslabón con procesos de demanda correlacionados en el tiempo y demuestra que es mejor invertir en la reducción de tiempos de reposición en un ambiente con este tipo de demandas.

Otros autores han abordado el tema de inventarios en la cadena de abastecimiento como una parte integral de modelos de programación lineal o no-lineal enteramixta aplicados a la optimización de cadenas de abastecimiento regionales e internacionales. Por ejemplo, Vidal (1998), Vidal y Goetschalckx (2001) y Goetschalckx et al. (2002), consideran los costos de inventarios en la función objetivo de los modelos matemáticos, como una aproximación proporcional a la demanda promedio. Cole (1995) introduce la consideración de demanda probabilística en centros de distribución en un modelo de programación lineal enteramixta y resuelve algunos casos de tamaño medio, identificando los inventarios máximos óptimos en un sistema de control periódico en la cadena. Por otra parte, Vidal y Goetschalckx (2000) consideran costos de inventarios en la función objetivo de un modelo de programación lineal entera-mixta, asumiendo demanda aproximadamente uniforme y tiempos de reposición aleatorios. Otros autores, como Sabri y Beamon (2000), combinan un submodelo estocástico operacional, el cual considera demanda y tiempos de reposición de producción y suministro estocásticos, con un submodelo estratégico determinístico.

Los sistemas reales de una bodega y $M$ detallistas se caracterizan por tener cientos de proveedores con comportamientos diferentes, cientos de miles de productos, detallistas muy diferentes entre sí y otras complejidades tales como distribuciones de demanda diferentes a la de Poisson, clasificación $A B C$ diferenciada por bodega y detallistas, el manejo de productos nuevos, los productos con demanda errática ó intermitente, entre muchas otras. Por lo tanto, la aplicación directa de los desarrollos anteriores es cuestionable y hace que deba pensarse en métodos heurísticos y prácticos de administración de inventarios, muy posiblemente con sistemas de control y de pronósticos "desarrollados en casa".

Además, como se ilustrará posteriormente, para el diseño de un sistema efectivo de administración y control de inventarios, no es necesario inicialmente considerar aspectos de costos de mantenimiento del inventario ni de ordenamiento o alistamiento. Esto se debe principalmente a que buenas técnicas de control implican directamente reducción de estos costos, sin necesidad de estimarlos previamente o de considerarlos dentro de la función objetivo de los modelos matemáticos.

Este trabajo de investigación aplicada pretende demostrar la utilidad del uso de técnicas cuantitativas para el control de inventarios en cadenas de abastecimiento con una bodega y $M$ detallistas. En la sección siguiente se presentan las principales características de los métodos utilizados en la aplicación. La sección 3 ilustra los resultados obtenidos mediante la aplicación de estos métodos en un sistema real de comercialización de productos de consumo masivo y, finalmente, la sección 4 detalla algunas conclusiones y oportunidades de investigación futura en el área.

\section{SISTEMAS IMPLEMENTADOS DE PRONÓSTICOS Y DE CONTROL DE INVENTARIOS}

Más que un desarrollo de control de inventarios puramente teórico, la metodología utilizada ha tenido en cuenta prácticamente todos los aspectos de la cadena de abastecimiento en forma integral. Esto se considera de importancia fundamental, ya que así se desarrolle el mejor modelo posible de control, si no se tienen en cuenta los aspectos administrativos, logísticos, 
financieros y de todo tipo presentes en toda la cadena de suministro y sus complejidades adicionales, los modelos tendrían pocas probabilidades de éxito, por mejores formulaciones que se utilicen. La literatura, en general, ignora este importante aspecto.

La Figura 2 muestra un diagrama causal que describe las principales interrelaciones entre los eslabones de la cadena de abastecimiento considerada. La gran interdependencia de las decisiones claramente afecta el funcionamiento global de la cadena de suministro. Por ejemplo, en algunas ocasiones se generan decisiones 'circulares', como el hecho de que el tiempo de reposición de un proveedor puede depender de la capacidad de recepción de la bodega y de la cantidad ordenada, la cual a su vez depende del tiempo de reposición del proveedor. Al parecer, la única forma práctica de resolver este problema ha sido por ensayo y error y se constituye en una pregunta abierta para investigación futura.

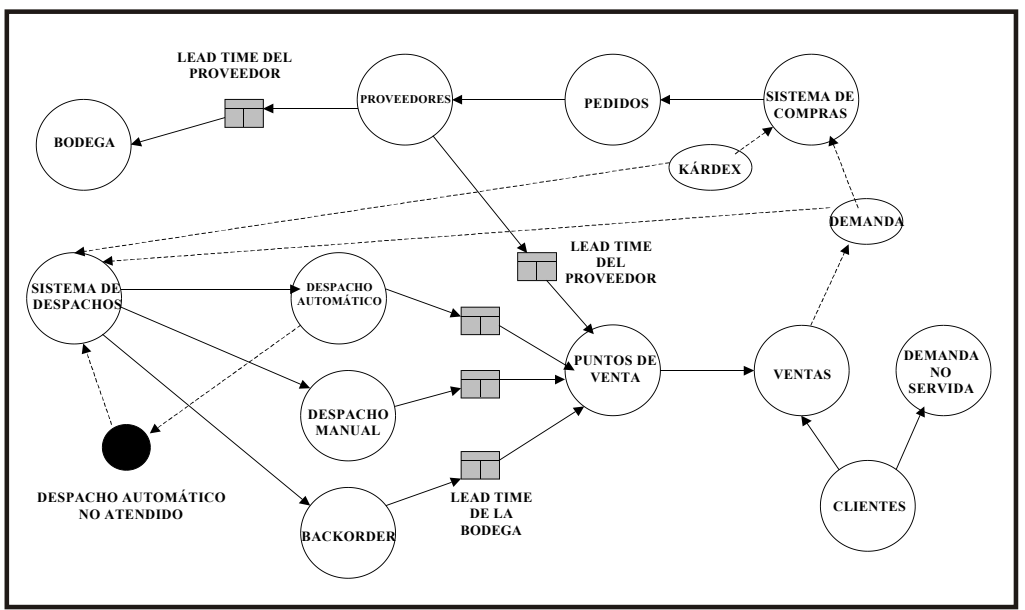

Figura 2. Diagrama causal de la cadena de abastecimiento considerada

El principal problema de un sistema de control de inventarios de una cadena de suministro con una bodega central y M puntos de venta es el de determinar dónde debe mantenerse el inventario, en qué cantidad y la coordinación de las decisiones sobre inventarios a lo largo de la cadena. De acuerdo con Silver et al. (1998), el inventario de seguridad es conveniente mantenerlo tanto en la bodega como en los puntos de venta. Específicamente, ellos sostienen que, "De los modelos básicos y de acuerdo con nuestras propias experiencias, podemos afirmar que es más atractivo mantener inventarios centralmente (refiriéndose a la bodega), cuando los costos de mantenimiento del inventario son apreciablemente menores allí y/o cuando los tiempos de reposición de distribución son relativamente pequeños ${ }^{4} . "$ Axsäter (2000), por el contrario, sostiene que los inventarios en los puntos de venta son necesarios para brindar un adecuado nivel de servicio. Afirma, además, que, "Un mayor stock en la bodega dará como resultado tiempos de reposición más cortos y menos variables. Sin embargo, la mejor distribución del inventario en todo el sistema dependerá de la estructura del mismo, de las variaciones de la demanda, de los tiempos de transporte y del valor de los productos. Existen situaciones para las cuales debería mantenerse un nivel de inventario relativamente alto en la bodega, pero es más común que la solución óptima signifique un nivel de inventario muy bajo en la bodega, mucho más bajo que lo que la mayoría de los analistas pudieran esperar" 5 .

Un método que ofrece algunas ventajas es el denominado sistema de control de stock base (BaseStock Control System), presentado por Silver et al. (1998), el cual supone Que en la cadena existe información global y control descentralizado. La información global se refiere a que, tanto en la bodega central como en los detallistas, se conoce la demanda

\footnotetext{
4 Silver et al. (1998), pág. 516.

5 Axsäter (2000), pág. 117
} 
externa de los consumidores en tiempo real y con ella se alimentan los pronósticos y se realiza el control de inventarios. El control descentralizado consiste en que las decisiones son tomadas por cada eslabón de la cadena independientemente, o sea, la bodega central y cada punto de venta en este caso.

Silver et al. sostienen que este sistema necesita de tecnología avanzada de información, tal como EDI para funcionar adecuadamente. La clave consiste en que las decisiones para emitir órdenes y mantener inventarios se toman en cada punto de venta y en la bodega central basadas en la información de la demanda real del consumidor final, en lugar de hacerlo en la bodega con base en la demanda inducida por los puntos de venta. Esta metodología se utiliza principalmente en sistemas de control continuo $(S, S)$ y de control periódico $(R, S)$, considerando el inventario efectivo definido como:

Inventario Efectivo = Inventario de Escalón + Órdenes Pendientes

Donde el Inventario de Escalón se define como el inventario que ha pasado por la bodega central (y ha sido despachado hacia los puntos de venta), pero que aún no ha sido comprometido con los clientes o consumidores finales. En el caso de un sistema continuo $(5,5)$, para el cálculo del inventario de seguridad se utiliza el tiempo de reposición que el escalón anterior presente. Por ejemplo, el tiempo de reposición que los puntos de venta utilizan para calcular sus inventarios de seguridad es la demora en que la bodega central incurre al alistar, despachar y transportar los pedidos, asumiendo que ésta siempre tiene los productos disponibles, o sea que el tiempo de reposición de los proveedores no afecta dicho cálculo. En un sistema de control periódico $(R, S)$, este tiempo de reposición debe incrementarse con el tiempo de revisión $R$ correspondiente de cada escalón.

Dada la disparidad de criterios y las complejidades descritas anteriormente, se diseñó un método híbrido, basado en información global, pero con decisiones tomadas centralmente. En otras palabras, las decisiones de compra de la bodega central y de reposición hacia los puntos de venta se toman centralizadamente, generando despachos automáticos hacia los puntos de venta. El sistema, sin embargo, es de tipo 'pull' porque la bodega mantiene inventario y no lo 'empuja' hacia los puntos de venta, y basa sus decisiones de compra en la demanda real observada en los detallistas.

Se escogió un sistema de control periódico $(R, 5)$, tanto en la bodega como en los puntos de venta, diferenciado por clase de producto A, B, C ó producto nuevo. Esta decisión fue motivada principalmente por los aspectos logísticos y administrativos de las compras en bodega y de los despachos a los detallistas y por la facilidad de coordinación en las decisiones de compra. Un aspecto importante es la distinción que debe hacerse en la clasificación de cada producto, dependiendo de la bodega central y de cada punto de venta. Por ejemplo, un producto puede ser clase $A$ en la bodega, que lo provee a todos los puntos de venta, pero puede ser B ó C en algunos puntos de venta donde no es tan demandado por los clientes. Esto induce otras complejidades en el diseño de los sistemas de pronósticos, y la necesidad de redefinir la clasificación $A B C$ en forma periódica.

Uno de los aspectos fundamentales que se tuvo que abordar en este proyecto fue la definición de las técnicas de pronósticos de demanda a implementar. Se resalta el hecho que siempre en el sistema se trata de pronosticar la demanda real y no las ventas, lo que se ha conseguido mediante la medición de la demanda no servida o no satisfecha en cada punto de venta. Se simularon diferentes sistemas de pronósticos tradicionales, tales como promedio móvil y suavización exponencial simple y doble, métodos auto-adaptivos y algunos métodos para productos con demanda errática, utilizando datos históricos disponibles. Este proceso consiste en 
utilizar parte de la historia para iniciar el sistema de pronósticos y simular el pronóstico para el resto de la historia con el fin de estudiar el comportamiento del mismo.

Mo se detectó estacionalidad en el tipo de productos manejados y por ello no fue necesario considerar métodos con factores estacionales. La implementación de los sistemas de pronósticos se hizo con desarrollos "hechos en casa", lo cual es una de las fortalezas de este proyecto. La Tabla 1 ilustra los sistemas de pronósticos y de control utilizados en el sistema de compras a proveedores.

Tabla 1. Sistemas de pronósticos y de control de inventarios utilizados en el sistema de compras a proveedores

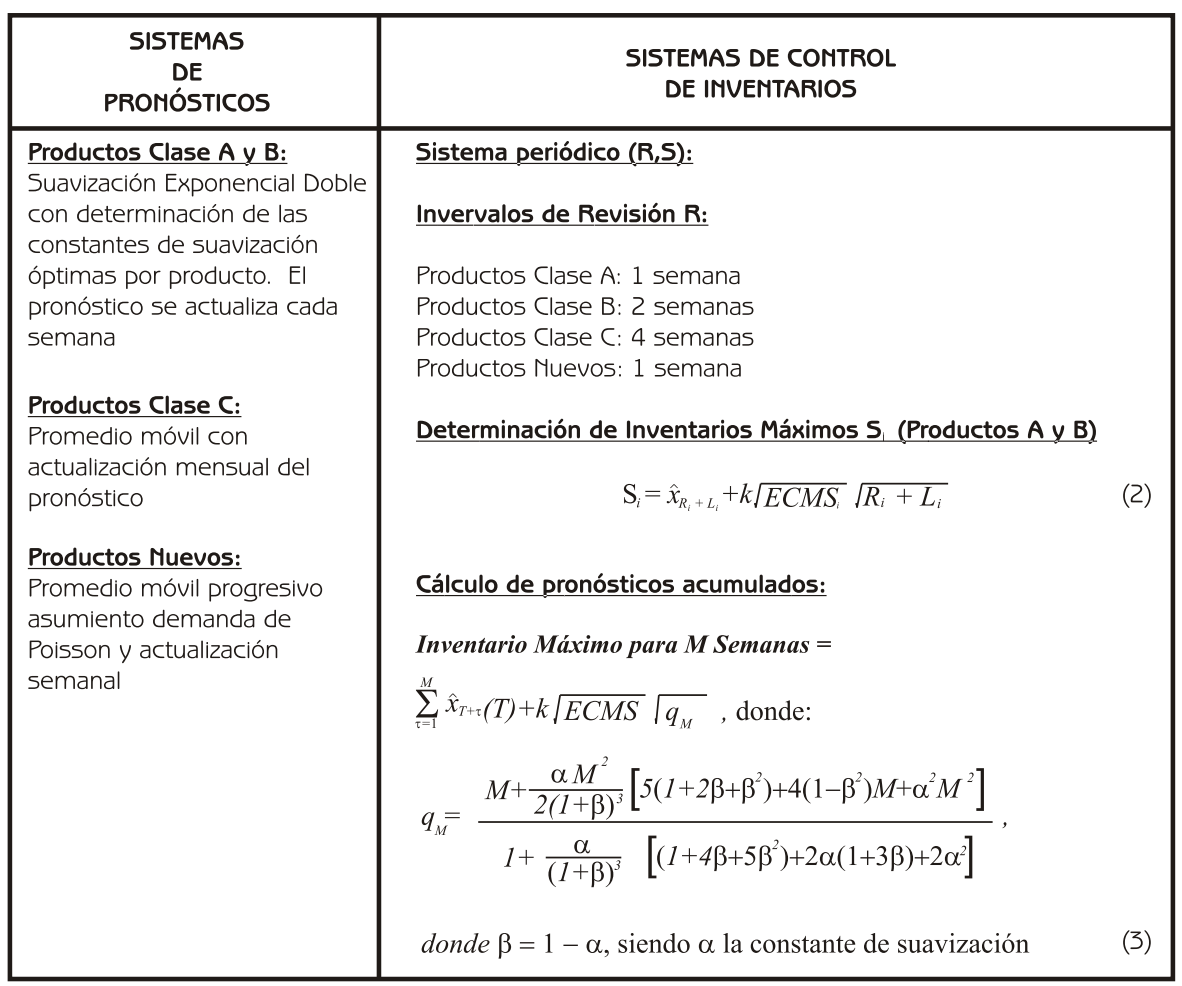

La ecuación (2) se usa para determinar los inventarios máximos de cada producto $i$ de clase A ó $B$, la cual utiliza errores cuadráticos medios suavizados, ECMS, permitiendo el cálculo dinámico de los inventarios de seguridad. Esta ecuación considera el tiempo de reposición de cada producto, $L_{i}$ y su intervalo de revisión $R_{i}$. El factor de seguridad, $k$, se determina de acuerdo con el nivel de servicio especificado para cada tipo de producto.

Es importante notar que los tiempos de reposición utilizados son los prometidos por cada proveedor, ya que se considera que el centro de distribución almacena inventario y no funciona como un centro de crossdocking, donde sí debería utilizarse la suma de los tiempos de reposición de los proveedores y del centro de distribución hacia los detallistas ${ }^{6}$. Además, este manejo permite controlar los problemas de temporalidad que se presentan, ya que a los proveedores se les revisa y ordena a lo largo de cada semana y a los puntos de venta se les despacha todos los productos a lo largo de una semana también.

Por otra parte, las ecuaciones (3) determinan los pronósticos acumulados para M períodos futuros, utilizando el error cuadrático medio suavizado en lugar de la desviación absoluta media suavizada, previendo cualquier caso de demanda no normal ${ }^{7}$. Estas ecuaciones han sido fundamentales para el establecimiento de los volúmenes adecuados de compra, especialmente cuando se hace necesario estimar la demanda para M períodos futuros o cuando se consideran negociaciones
6 Observar, por ejemplo, Simchi-Levi et al. (2003), pág. 6768.

7 Estas ecuaciones fueron adaptadas de Montgomery et al. (1990), pág. 210212.

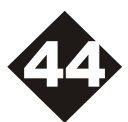

VOLUMEN 6 - No. 1 - Septiembre de 2004 
especiales con los proveedores.

Los sistemas de pronósticos y de control utilizados para el despacho automático del centro de distribución a los detallistas son semejantes a los de compras, ajustados con los tiempos de reposición adecuados para cada caso. Dada la diferencia existente entre las variabilidades de los casos individuales de demanda en cada detallista y la variabilidad de la demanda acumulada de cada producto, se decidió utilizar un mayor factor de seguridad para los sistemas de control de compras. El parámetro de diseño es de un riesgo de faltantes por cada ciclo de reposición del 1\% para compras y del $2.5 \%$ para el sistema de despacho automático. La correspondencia de este nivel de servicio especificado se ha podido comprobar con los resultados reales medidos en la práctica.

Por otra parte, para el caso de cada producto nuevo $i$, se ha considerado demanda de Poisson, con la siguiente ecuación para la determinación de los inventarios máximos:

$\mathrm{S}_{i}=\hat{x}_{R_{i}+L_{i}}+k \sqrt{\hat{x}_{R_{i}+L_{i}}} \sqrt{R_{i}+L_{i}}$

Esta ecuación se aplica tanto en el sistema de despacho automático, como en el sistema de compras, ajustada con los tiempos de reposición correspondientes para cada caso. A medida que la demanda de cada producto nuevo va creciendo, se incluye más información en el modelo, hasta que el producto se cambia de clasificación ( $A$, B ó C) y sus sistemas de pronóstico y de control son administrados por los correspondientes de cada clase de producto. El método de control de productos nuevos ha sido fundamental para el manejo de los inventarios de nuevos detallistas que se fueron creando durante el desarrollo del proyecto.

Adicionalmente, los sistemas de compras y despacho automático generan señales de rastreo con base en la desviación absoluta media suavizada y calculan automáticamente las constantes de suavización óptimas en forma periódica, por cada producto y por cada localidad. Las señales de rastreo son una herramienta muy útil para evaluar el comportamiento del sistema de pronósticos ante cambios inesperados de demanda, y envían una señal de alarma, bien para la redefinición de los parámetros del modelo, como por ejemplo la constante de suavización, y/o para la revisión más detallada del fenómeno de demanda por parte del personal que administra los inventarios. Igualmente existe un sistema de control de datos atípicos, tanto para la iniciación de los pronósticos como para el control dinámico del inventario.

La variabilidad de los tiempos de reposición de proveedores a bodega central no se ha modelado aún en forma directa, debido a limitaciones de información y a complejidades tales como el cumplimiento parcial de órdenes de compra de parte de los proveedores. Para reaccionar a estas fluctuaciones, se han establecido inventarios adicionales de seguridad. Específicamente, se ha dejado de considerar parte del inventario de escalón detallado en la expresión (1) para el cálculo del sugerido de compra. Esto deja posibilidades futuras de mejoramiento en los sistemas de control implementados, cuando se disponga de la información necesaria. Muestra investigación actual está dirigida hacia la implementación gradual de la expresión (1) en todos los eslabones de la cadena, definiendo una metodología para involucrar explícitamente la variabilidad de los tiempos de reposición de los proveedores en los análisis, a través de modelos estocásticos compuestos.

Mo fue necesario estimar a priori los costos de ordenamiento, de mantenimiento del inventario y de faltantes, para poner en marcha los sistemas de control, como sería el caso de gran parte de los métodos observados en la literatura. Lo que los autores se proponen hacer es tratar de estimar estos costos a posteriori con el objeto de verificar la bondad de los sistemas de control y compararlos con posibles técnicas adicionales de 
refinamiento, especialmente para el caso de productos clase A. Este aspecto se considera muy importante pues facilita las aplicaciones en otros sistemas sin la necesidad inicial expresa de estimar dichos costos, lo cual es muy difícil de lograr con precisión en la mayoría de los casos. Además, la mayoría de los sistemas de inventarios son muy robustos en cuanto a cambios en los costos mencionados.

A manera de comprobación de la metodología utilizada, de acuerdo con Sanders y Manrodt (2003), de 240 compañías estadounidenses encuestadas, 48\% utilizan hojas electrónicas para hacer sus pronósticos, mientras que tan sólo el $10.8 \%$ de las mismas reportaron el uso de software comercial de pronósticos. Además, el $60 \%$ de las mismas dijeron estar insatisfechas con el comportamiento de su software de pronósticos. Las conclusiones más importantes de este estudio son, por una parte, que las empresas que utilizan procesos más formales de pronósticos obtienen mejores resultados, y, por otra parte, que la más probable causa de insatisfacción con los sistemas de pronósticos es la dificultad para comprender los resultados y leer los reportes.

Lo anterior sugiere que una muy buena opción es la implementación de técnicas de pronósticos formales desarrollados con base en el sistema de información propio de la empresa. Esta metodología se considera de punta porque aprovecha el sistema de información local de la empresa y utiliza modelos matemáticos y estadísticos de control, con lo que prácticamente se convierte en un sistema de planeación avanzada (APS) diseñado a la medida.

Finalmente, la gran cantidad de decisiones administrativas y de casos especiales presentes en un sistema de esta naturaleza, hace que la implementación y refinamiento de los modelos sean muy complejos. Para facilitar estas actividades, se desarrolló lo que se denominó la "carta de navegación", la cual describe en forma precisa todas las actividades necesarias en toda la cadena de suministro que deben llevarse a cabo para garantizar el correcto funcionamiento de los modelos matemáticos y estadísticos implementados.

\section{RESULTADOS}

Se aplicó la metodología descrita a un caso real de una cadena de suministro de productos de consumo masivo, la cual se suple de más de 200 proveedores desde una bodega central que hace despachos de más de 6.000 productos hacia 34 puntos de venta, lo que ocasiona el diseño de un control real de cerca de 120.000 productos con condiciones de demanda diferentes, de acuerdo con el surtido tipo de cada detallista. Los principales resultados cuantitativos de este proyecto se muestran en la Tabla 2, la cual presenta el cambio porcentual del promedio y de la desviación estándar del inventario total, la rotación del inventario, el inventario pagado, el porcentaje de inventario pagado y la rotación neta del inventario. Dicho promedio y desviación estándar fueron determinados con base en los indicadores mensuales de cada año, antes y después de la implementación de los sistemas de pronósticos y control de inventarios diseñados. La reducción en la desviación estándar de cada indicador corrobora la estabilidad de los sistemas de control y de sus resultados a lo largo del tiempo.

Es importante notar que los indicadores mostrados en la Tabla 2 han venido presentando reducciones mes a mes, comparados en años sucesivos, desde la implementación del proyecto. Se ilustra el promedio de dichas reducciones, comparando 2003 contra 2000 (ó 2001 en algunas ocasiones, pues algunos modelos fueron implementados a mediados de este año).

Debe destacarse igualmente que durante el desarrollo del proyecto, se crearon 13 detallistas nuevos, lo que representa un incremento del 62 
por ciento, hecho que supuestamente debería haber aumentado el inventario total en la cadena. Por el contrario, la Tabla 2 muestra una disminución del inventario total en la cadena de suministro, evidenciando la extraordinaria capacidad de las técnicas cuantitativas aplicadas. Más importante aún, como consecuencia del balanceo de los inventarios, se mantuvo un nivel de servicio cercano al 98\%, lo que hace más significativo el resultado de los indicadores mostrados $^{8}$.

Tabla 2. Cambios en el promedio y desviación estándar de indicadores claves de la cadena de abastecimiento bajo estudio

\begin{tabular}{|l|l|l|}
\hline \multicolumn{1}{|c|}{ Indicador } & Promedio & $\begin{array}{c}\text { Desviación } \\
\text { Estándar }\end{array}$ \\
Inventario total a \$ de 2000 & $-9.81 \%$ & $-39.05 \%$ \\
Rotación de inventario (días) & $-8.31 \%$ & $-21.27 \%$ \\
Inventario pagado a \$ de 2000 & $-56.91 \%$ & $-33.27 \%$ \\
Porcentaje de inventario pagado & $-48.78 \%$ & $-26.16 \%$ \\
Rotación neta del inventario (días) & $-54.75 \%$ & $-30.96 \%$ \\
\hline
\end{tabular}

Debe destacarse igualmente que, durante el desarrollo del proyecto, se crearon 13 detallistas nuevos, lo que representa un incremento del 62 por ciento, hecho que supuestamente debería haber aumentado el inventario total en la cadena. Por el contrario, la Tabla 2 muestra una disminución del inventario total en la cadena de suministro, evidenciando la extraordinaria capacidad de las técnicas cuantitativas aplicadas. Más importante aún, como consecuencia del balanceo de los inventarios, se mantuvo un nivel de servicio cercano al $98 \%$, lo que hace más significativo el resultado de los indicadores mostrados.

La reducción de la rotación neta y del porcentaje del inventario pagado son los resultados más significativos, pues la organización vende la

8 Este nivel de servicio se calcula como la proporción de la demanda satisfecha directamente del inventario a la mano (fill rate) mayoría de sus inventarios antes de tener que pagarlos al proveedor, ya que los modelos permiten realizar la compra justa sin incurrir en excesos ni en faltantes frecuentes. Las figuras 3 a 5 muestran el comportamiento de indicadores claves a lo largo del horizonte del proyecto. Para garantizar la confidencialidad, las gráficas presentan cada indicador en escala relativa, siendo 1 el valor máximo observado.

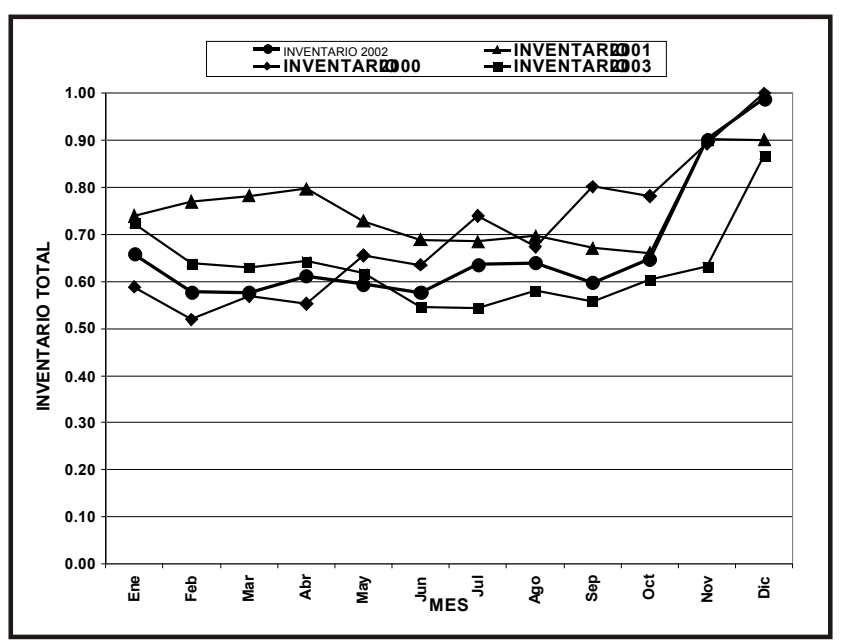

Figura 3. Inventario total a pesos constantes de 2000 (En escala relativa)

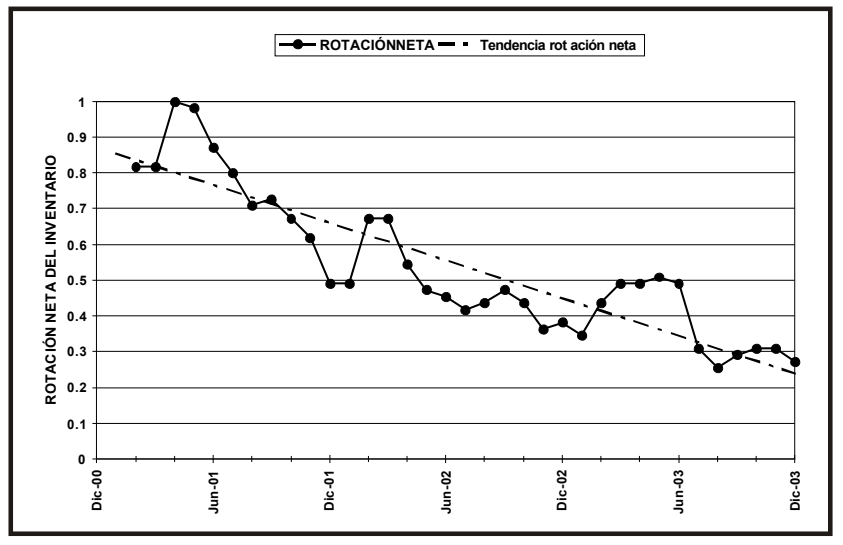

Figura 4. Rotación neta del inventario en días (En escala relativa)

La figura 3 muestra el comportamiento del inventario entre 2000 y 2003, a pesos constantes del año 2000. Obsérvese cómo el inventario total ha venido decreciendo mes a mes en forma casi continua desde la implementación 
completa de los modelos de control en Junio de 2001, a pesar de que, como se dijo anteriormente, se abrieron 13 detallistas nuevos entre Junio de 2000, fecha de iniciación del proyecto, y Diciembre de 2003.

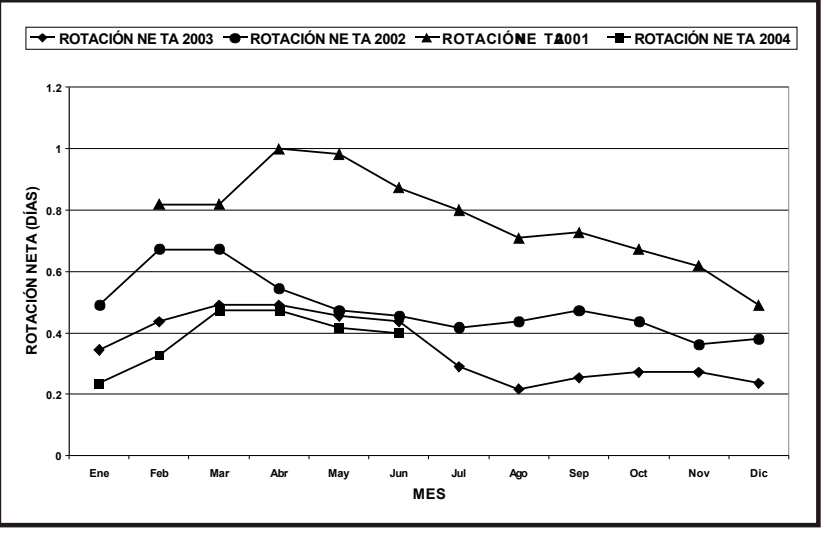

Figura 5. Rotación neta del inventario en días (Por año, en escala relativa)

En la figura 4 se muestra la disminución real que ha tenido la rotación neta del inventario en días, entre Febrero de 2001 y Diciembre de 2003. Este resultado demuestra que actualmente la empresa está comprando lo justo de acuerdo con su demanda y ha logrado establecer políticas transparentes de negociación con sus proveedores. La figura 5 muestra este mismo resultado con datos más recientes, evidenciando la continuidad del mejoramiento del indicador hasta Junio de 2004.

Otros resultados importantes de este proyecto han sido los siguientes:

■ La reducción de inventarios y el mantenimiento del nivel de servicio se logró conservando el mismo recurso en la bodega central, incluso con la apertura de los nuevos detallistas, al proponerse e implementarse la reducción de frecuencia de despachos hacia los detallistas.

च El carácter dinámico de los modelos de control ha permitido que las decisiones de ajuste y control sean administradas básicamente por los mismos modelos.

$\square$ El modelo de compras en el centro de distribución se ha convertido en una poderosa herramienta de negociación con los proveedores, evitando excesos de inventarios que se presentaban usualmente.

$\square$ Aunque la rotación total del inventario en días disminuyó en alrededor de un $8.3 \%$ en promedio, su trascendencia ha disminuido debido al aumento de importancia de la rotación neta, la cual, por consenso, es el mejor indicador del estado real de los inventarios de la empresa. Sin embargo, en los últimos meses se ha observado también un equilibrio en la rotación propiamente dicha y se ha llegado a la conclusión de que ambos indicadores pueden disminuirse en forma simultánea cuando se tenga una mejor colaboración de parte de los proveedores y se ejecuten los planes de mejoramiento adicional propuestos.

$\square$ Se ha estandarizado la depuración del inventario en la bodega central y en los puntos de venta, lo que ha eliminado los excesos de productos de lento o nulo movimiento y ha facilitado la determinación del surtido tipo en cada detallista.

$\square$ Se ha logrado capacitar al personal de la organización en el área de gestión y control de inventarios, lo que ha facilitado significativamente los resultados obtenidos.

Se destaca finalmente el logro de la estabilidad administrativa de la cadena de abastecimiento en forma integral. La implementación de los modelos de control de inventarios, en concordancia con las prácticas administrativas de la empresa, han permitido una integración de las decisiones a todo nivel, incluyendo la bodega central, el departamento de compras y mercadeo, el departamento de sistemas, los puntos de venta y la administración central. Este resultado ha sido de gran importancia, pues se estaba buscando algo semejante desde mucho tiempo atrás. La 'carta de navegación' ha jugado un papel fundamental para este efecto. 
Lo más importante de los resultados anteriores es que esta tendencia ha continuado durante el primer semestre de 2004 y se han estabilizado gradualmente todos los sistemas de control, brindando al sistema un equilibrio que nunca había tenido anteriormente. Por esta razón, se espera que los resultados reales del proyecto superen en corto tiempo a lo reportado en esta publicación, ya que además se están investigando alternativas adicionales de mejoramiento de los sistemas de control y de los procesos a lo largo de toda la cadena, las cuales se describen en la sección siguiente.

\section{CONCLUSIONES E INVESTIGACIÓN FUTURA EN EL ÁREA}

Con la implementación "en casa" de técnicas relativamente sencillas de pronósticos y de control de inventarios, se lograron resultados significativos en una cadena de suministro de productos de consumo masivo con una bodega central surtida por cerca de 200 proveedores, la cual atiende a 34 detallistas, con un número total de productos de alrededor de 120.000. Se implementaron modelos de control de inventario periódico $(R, S)$ para las compras en la bodega central y para los despachos automáticos hacia los detallistas. Fueron utilizados sistemas de pronósticos de demanda tradicionales, tales como promedio móvil y suavización exponencial doble, refinados con técnicas de detección de problemas mediante señales de rastreo y eliminación de datos atípicos de demanda. Se diseñaron además sistemas especiales de control para productos nuevos en la cadena.

Con los modelos implementados se logró reducir el inventario total en promedio en un $10 \%$, a pesar del incremento en el número de detallistas de un $62 \%$, mejorando el nivel de servicio real al cliente, el cual se ha mantenido alrededor del 98\%; se logró reducir el promedio del inventario pagado a proveedores en un 57\% y el promedio de la rotación neta en días de inventario pagado en un $55 \%$, creando una situación de estabilidad de los modelos de control y de "tranquilidad administrativa", buscadas por la empresa por varios años antes de aplicar modelos de decisión cuantitativos. La tendencia de mejoramiento se ha mantenido hasta el primer semestre de 2004.

En esta aplicación se destaca la utilización de conocidos sistemas de pronósticos y de control y de otros adaptados a la situación específica, como el caso de la predicción de demanda de productos nuevos y el cálculo dinámico de pronósticos acumulados, en un sistema extremadamente complejo. A pesar de que la literatura acerca del problema de una bodega y $M$ detallistas es muy amplia, no es clara su aplicación directa en sistemas reales de esta naturaleza, los cuales presentan dificultades técnicas y administrativas para la implementación de técnicas cuantitativas. Es por ello que este proyecto se considera pionero en la región, como un aporte de investigación aplicada para el mejoramiento de la competitividad de las organizaciones regionales.

Un aspecto para puntualizar es la importancia que tienen los procesos de colaboración en la cadena de abastecimiento. Por ejemplo, para los proveedores, quienes a su vez tienen su propia cadena de suministro de materias primas, sería muy interesante disponer de datos de demanda real de los consumidores y así disminuir el efecto látigo. Si esta práctica se generalizara a los clientes más importantes de cada proveedor, éste podría pronosticar su demanda con mejor precisión, evitando así faltantes de inventario que comúnmente se presentan y que causan un gran problema al romperse el inventario en la bodega central ocasionando el deterioro en el servicio al cliente. Este proceso de colaboración se constituiría evidentemente en una relación gana gana si se logran superar las dificultades de confianza y confidencialidad, barreras más prominentes de este tipo de relaciones.

Queda abierta también la pregunta de la conveniencia de la adopción de sofisticados 
sistemas de información, planeación y administración de la cadena de abastecimiento. Muchas veces estos se convierten en costosos sistemas de información solamente, sin que se puedan explotar todas sus posibilidades. Específicamente, en el área de inventarios, en general estos sistemas no son utilizados adecuadamente, y sus capacidades de pronósticos y de control no son aprovechadas en todo su potencial. En muchas ocasiones, como en el caso mostrado en esta publicación, sistemas sencillos hechos en casa pueden ser la respuesta rápida, parcial o definitiva, a los serios problemas de inventarios que presentan las organizaciones locales y nacionales en general.

Como investigación futura se han planteado diversos aspectos de importancia, tales como los siguientes, entre otros posibles:

$\square$ El análisis de las complejas relaciones que existen entre la determinación de los sugeridos de compra y la capacidad de recepción de la bodega central.

■ El análisis detallado de los productos de demanda errática a través de métodos de pronósticos más sofisticados y/o de acciones administrativas, tales como la consolidación de estos productos en la bodega central y/o en puntos clave de la cadena de suministro de la empresa.

■ La aplicación gradual de la expresión (1) para el cálculo de los sugeridos de compra en la bodega central, introduciendo la variabilidad del tiempo de reposición de los proveedores en los cálculos de los inventarios de seguridad.

■ La estimación de los costos de inventarios generados por las nuevas prácticas de control de inventarios, su comparación con los costos anteriores y su establecimiento como indicadores de eficiencia para evaluar el comportamiento de futuros métodos de control.

$\square$ La prueba de otros sistemas de control de inventarios más sofisticados, tales como los
$(R, s, 5)$, especialmente para los productos clase $A$.

■ La integración de los procesos de control y gestión de inventarios con otros procesos de la cadena, como por ejemplo los sistemas de transporte, incluyendo las técnicas de ruteo y de selección del modo de transporte.

$\square$ El refinamiento las actividades administrativas y de control a lo largo de toda la cadena de abastecimiento.

■ La aplicación de estos métodos en otros departamentos de la empresa que involucren, por ejemplo, casos de demanda estacional y posibles demandas correlacionadas en el tiempo.

■ El análisis de posibles alianzas estratégicas con cadenas de suministro semejantes.

$\square \quad$ La aplicación de técnicas semejantes en ambientes productivos donde la situación del tipo de proceso de producción y la demanda dependiente crean complejidades adicionales.

Desde el punto de vista de la Investigación de Operaciones, este proyecto muestra cómo la aplicación real de técnicas relativamente sencillas produce resultados significativos y de importancia fundamental para las organizaciones, lo que puede volverlas más competitivas y mejor preparadas para el proceso de globalización que está en marcha. La adopción y aplicación de técnicas cuantitativas como herramientas fundamentales para los procesos de toma de decisiones a todo nivel en cualquier empresa de naturaleza industrial, comercial o de servicios no son una sofisticación innecesaria, costosa y de difícil comprensión; por el contrario, son una necesidad.

\section{RECONOCIMIENTOS}

Esta investigación ha sido financiada parcialmente por el Instituto Colombiano para el Desarrollo de la Ciencia y la Tecnología "Francisco José de Caldas" (COLCIEMCIAS), por la 
UMIVERSIDAD DEL VALLE y por la Caja de Compensación Familiar del Valle del Cauca (COMFAMDI). Se agradece la valiosa labor desarrollada por Jorge Enrique Restrepo y Arnulfo Zamora para la implementación de los modelos de pronósticos y control de inventarios, y de todo el personal involucrado directa o indirectamente con este proyecto.

Los autores agradecen las valiosas recomendaciones de dos evaluadores anónimos para el mejoramiento del contenido y presentación de este artículo.

\section{REFERENCIAS}

1. Axsäter, S. (1993a), "Continuous Review Policies for MultiLevel Inventory Systems With Stochastic Demand", En: S. Graves, A. Rinnooy Kan y P. H. Zipkin (Editores), Logistics of Production and Inventory, Vol. 4, Amsterdam, Elsevier (MorthHolland).

2. Axsäter, S. (1993b), "Exact and Approximate Evaluation of BatchOrdering Policies for TwoLevel Inventory Systems", Operations Research 41, 77785.

3. Axsäter, 5. (1998), "Evaluation of Installation Stock Based $(R, Q)$-Policies for Two-Level Inventory Systems with Poisson Demand", Operations Research 46, Supl. Mo. 3, 51355145.

4. Axsäter, 5. (2000), Inventory Control, Kluwer Academic Publishers, Boston.

5. Axsäter, S., J. Marklund y E. A. Silver (2002), "Heuristic Methods for Centralized Control of One-Warehouse, M-Retailer Inventory Systems", Manufacturing \& Service Operations Management 4 (1), 7597.

6. Chen, F. y R. Samroengraja (2000), "A Staggered Ordering Policy for OneWarehouse, Multiretailer Systems", Operations Research 48 (2), 281293.

7. Cheng, F. y Y. Zheng (1997), "OneWarehouse MultiRetailer Systems With Decentralized Stock Information", Operations Research 45 (2), 27587.
8. Clark, A. J. y H. Scarf (1960), "Optimal Policies for a MultiEchelon Inventory Problem", Management Science 6 (4), 47590.

9. Cohen, M., P. Kleindorfer y H. Lee (1986), "Optimal Stocking Policies for Low Usage Items in MultiEchelon Inventory Systems", Maval Research Logistics 33, 1738.

10.Cole, M. H. (1995), Service Considerations and the Design of Strategic Distribution Systems, Disertación Doctoral, School of Industrial and Systems Engineering, Georgia Institute of Technology, Atlanta, Georgia, USA.

11.Dong, L. y H. L. Lee (2003), "Optimal Policies and Approximations for a Serial Multiechelon Inventory System With Time Correlated Demand", Operations Research 51 (6), 969980.

12.Federguen, A. y P. Zipkin (1984), "Allocation Policies and Cost Approximations for Multilocation Inventory Systems, Maval Research Logistics 31, 97129.

13.Forsberg, R. (1995), "Optimization of Orderup-to-S Policies for Two-Level Inventory Systems with Compound Poisson Demand", European Journal of Operational Research 81, 14353.

14.Forsberg, R. (1996), "Exact Evaluation of ( $R$, Q)-Policies for Two-Level Inventory Systems with Poisson Demand", European Journal of Operational Research 96, 13038.

15. Goetschalckx, Marc, C. J. Vidal y K. Dogan (2002), "Modeling and design of global logistics systems: A review of integrated strategic and tactical models and design algorithms", European Journal of Operational Research 143 (1), 118.

16.Graves, S. C. (1996), "A Multiechelon Inventory Model with Fixed Replenishment Intervals", Management Science 42 (1), 118.

17. Lee, H., P. Padmanabhan y S. Whang (1997), "Information Distortion in a Supply Chain: The Bullwhip Effect", Management Science 43 (4), 54658.

18.Lee, H., K. C. So y C. S. Tang (2000), "The Value of Information Sharing in a Two-Level Supply Chain", Management Science 46 (5), 626643. 
19.Lu, L. y M. Posner (1994), "Approximation Procedures for the OneWarehouse, MultiRetailer System", Management Science 40 (10), 130516.

20.Matta, K. F. y D. Sinha (1995), "Policy and Cost Approximations of TwoEchelon Distribution Systems with a Procurement Cost at the Higher Echelon", IIE Transactions 27, 63845.

21.Moinzadeh, K. (2002), "A Multi-Echelon Inventory System with Information Exchange", Management Science 48 (3), 414426.

22. Montgomery, D. C., L. A. Johnson y J. S. Gardiner, Forecasting and Time Series Analysis, McGraw-Hill, Inc., Mew York, 1990.

23. Muckstadt, J. A. y R. O. Roundy (1987), "Multiltem, OneWarehouse, MultiRetailer Distribution Systems", Management Science 33 (12), 161321.

24.Özer, Özalp (2003), "Replenishment Strategies for Distribution Systems Under Advance Demand Information", Management Science 49 (3), 255272.

25.Sabri, E. H. y B. M. Beamon (2000), "A MultiObjective Approach to Simultaneous Strategic and Operational Planning in Supply Chain Design", Omega 28, 581598.

26.Sanders, M. R. y K. B. Manrodt (2003), "Forecasting Software in Practice: Use, Satisfaction and Performance", Interfaces 33 (5), 9093.

27.Schwarz, L. B. (1973), "A Simple Continuous Review Deterministic OneWarehouse MRetailer Inventory Problem", Management Science 19 (5), 55566.

28.Sherbrooke, C. C. (1968), "METRIC A MultiEchelon Technique for Recoverable Item Control", Operations Research 16 (1), 10321.

29.Silver, Edward A., David F. Pyke y Rein Peterson (1998), Inventory Management and Production Planning and Scheduling, tercera edición, John Wiley \& Sons, Mew York.

30.Simchi-Levi, D., P. Kaminsky y E. Simchi-Levi, Designing and Managing the Supply Chain: Concepts, Strategies, and Case Studies,
Segunda Edición, McGraw-Hill Irwin, Boston, 2003.

31.Vidal, C. J. (1998), "A Global Supply Chain Model with Transfer Pricing and Transportation Cost Allocation," Disertación Doctoral, School of Industrial and Systems Engineering, Georgia Institute of Technology, Atlanta, Georgia.

32.Vidal, C. J. (2001), "El desbalanceo de inventarios: Un problema muy común", Diario La República, Moviembre.

33.Vidal, C. J. (2002), "Las complejas decisiones en el manejo de los inventarios", Editorial, Revista Zonalogística 2 (8), 4.

34.Vidal, C. J. y M. Goetschalckx (2000), "Modeling the Impact of Uncertainties on Global Logistics Systems," Journal of Business Logistics 21 (1), 95120.

35.Vidal C. J. y M. Goetschalckx (2001), "A Global Supply Chain Model with Transfer Pricing and Transportation Cost Allocation," European Journal of Operational Research 129, 13458. 